\title{
Topology Induced Macroscopic Quantum Coherence in Josephson Junction Networks
}

\author{
G. Giusiano, ${ }^{1}$ F. P. Mancini, ${ }^{1}$ P. Sodano, ${ }^{1}$ and A. Trombettoni ${ }^{2}$ \\ ${ }^{1}$ Dipartimento di Fisica and Sezione I.N.F.N., Università di Perugia, \\ Via A. Pascoli Perugia, I-06123, Italy \\ ${ }^{2}$ I.N.F.M. and Dipartimento di Fisica, Università di Parma, \\ parco Area delle Scienze 7 A Parma, I-43100, Italy
}

(Dated: January 3, 2019)

\begin{abstract}
We argue that Josephson junction networks may be engineered to allow for the emergence of new and robust quantum coherent states. We provide a rather intuitive argument showing how the change in topology may affect the quantum properties of a bosonic particle hopping on a network. As a paradigmatic example, we analyze in detail the quantum and thermodynamic properties of non-interacting bosons hopping on a comb graph. We show how to explicitly compute the inhomogeneities in the distribution of bosons along the comb's fingers, evidencing the effects of the topology induced spatial Bose-Einstein condensation characteristic of the system. We propose an experiment enabling to detect the spatial Bose-Einstein condensation for Josephson networks built on comb graphs.
\end{abstract}

\section{INTRODUCTION}

Quite recently it has been evidenced that quantum states may support new kind of orders, which cannot be characterized by broken symmetries and, thus, cannot be described by the conventional Ginzburg-Landau theory 1]. Quantum orders may be viewed as the pertinent description of the pattern of the quantum entanglement in a quantum many-body ground-state. Quantum or topological orders have already been extensively used in the analysis of Fractional Quantum Hall systems 2], leading to an elegant explanation of their robust (against a weak but, otherwise, arbitrary perturbation) ground-state degeneracy and evidencing the intimate connection between the ground-state and the statistics of quasi-particles. The robustness of the ground-state degeneracy makes quantum ordered states very relevant candidates for the engineering of quantum devices naturally taming the intrinsic decoherence of other solid state devices [3]. Very recent studies hint to the new and exciting possibility that the topology of Josephson junction networks (JJN) may be crucial for inducing novel and unexpected macroscopic quantum phenomena [4] as well as for opening 5 to the possibility for an explicit realization of a quantum ordered state.

In this paper we shall focus our attention on the recently discovered possibility that JJN built on suitable graphs may support new and interesting quantum macroscopic states induced only by a pertinent engineering of the geometry and topology of the graph supporting the network [4, 6]. In particular, we shall consider JJN built on comb-like graphs, evidencing the fact that - already for this very simple graph topology - the array exhibits quantum macroscopic coherence at low temperatures. After discussing the theoretical possibility of a spatial Bose-Einstein condensation (BEC) of Cooper pairs for a JJN built on a comb-graph, we shall propose here a simple experiment which could enable to observe BEC in these systems.

A network of superconducting classical Josephson junctions is usually described by the Hamiltonian [7]:

$$
H_{J J}=-J_{0} \sum_{\left\langle x, y ; x^{\prime}, y^{\prime}\right\rangle} \cos \left(\phi_{x, y}-\phi_{x^{\prime}, y^{\prime}}\right) \text {. }
$$

On each site of the network there is a superconducting grain and the junctions are located between neighboring sites with Josephson energy $J_{0} ; \phi_{x, y}$ is the phase of the superconducting order parameter at site labeled by $(x, y)$. The parameter $J_{0}$ is connected to the critical Josephson current $I_{c}$ by the relation $I_{c}=2 e J_{0} / \hbar\left[\right.$ ] . The sum $\left\langle x, y ; x^{\prime}, y^{\prime}\right\rangle$ runs over all the distinct nearest-neighbors pairs. The Hamiltonian (1) is closely related to the Hamiltonian describing non-interacting bosons hopping on a generic network

$$
H=-t \sum_{\left\langle x, y ; x^{\prime}, y^{\prime}\right\rangle}\left(\hat{a}_{x, y}^{\dagger} \hat{a}_{x^{\prime}, y^{\prime}}+H . C .\right) .
$$

In Eq. (2), $t$ is the hopping parameter while $\hat{a}_{x, y}^{\dagger}\left(\hat{a}_{x, y}\right)$ is the creation (annihilation) operator for bosons; $\hat{n}_{x, y}=$ $\hat{a}_{x, y}^{\dagger} \hat{a}_{x, y}$ is the number operator at site $(x, y)$. The filling, i.e., the average number of particles per site, is defined as $f=N_{T} / L^{2}$, where $N_{T}$ is the total number of bosons and $L^{2}$ is the number of sites. When $f \gg 1$ and the fluctuations of the particle numbers per site are much smaller than $f$, one can safely substitute the operator $\hat{a}_{x, y}$ with $\sqrt{N_{x, y}} e^{i \phi_{x, y}}$, where $N_{x, y}$ is the number of particles at the site $(x, y)[9$, 10]. As a result, the Hamiltonians (10) and (2) may be 


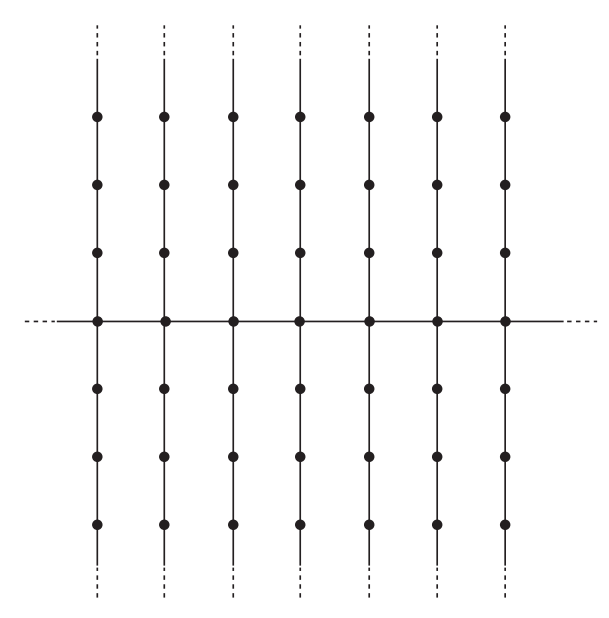

FIG. 1: Comb graph.

regarded as equivalent, provided that

$$
J_{0} \approx 2 t \sqrt{N_{x, y} N_{x^{\prime}, y^{\prime}}} \approx 2 t f .
$$

The plan of the paper is the following: in Sec. II we analyze the spectrum of bosons hopping on a comb-shaped graph. There we shall also use a variational approach aimed to elucidate how a mere change in the network's topology is crucial for explaining the emergence of localized states in the single-particle spectrum of free bosons hopping on a pertinent graph. In Sec. III we analyze the thermodynamic properties of non-interacting bosons hopping on a comb-graph: we compute the critical temperature $T_{c}$ and the inhomogeneous spatial distribution of bosons over the array as a function of the scaled temperature $T / T_{c}$. In Sec. IV we propose a method to detect BEC in a comb-shaped JJN: for this purpose we shall determine the Josephson critical current $I_{c}$ as a function of the scaled temperature $T / T_{c}$ and of the location of the junction.

\section{TOPOLOGY INDUCED LOCALIZATION FOR BOSONS HOPPING ON A GRAPH}

In this section, we shall illustrate how a mere change in the topology of a lattice may affect the spectrum of a quantum mechanical system by analyzing the properties of non-interacting bosons hopping on a comb-shaped network.

A comb graph (see Fig. (1) is composed of one-dimensional chains (fingers) grafted periodically on a linear chain (backbone). Each site of the comb can be naturally labeled introducing two integer indices $(x, y)$, where $x$ labels the different fingers and $y$ represents the distance from the backbone. Each site on the finger is linked to two neighbors whereas each site of the backbone has four neighbors. The Hamiltonian (2) on a generic graph can be written as:

$$
H=-t \sum_{x, y ; x^{\prime}, y^{\prime}} A_{x, y ; x^{\prime}, y^{\prime}} \hat{a}_{x, y}^{\dagger} \hat{a}_{x^{\prime}, y^{\prime}} .
$$

The topology of the network is fully described by the adjacency matrix $A_{x, y ; x^{\prime}, y^{\prime}}$ which is equal to 1 if $\left(x, y ; x^{\prime}, y^{\prime}\right)$ is a link and 0 otherwise. The single-particle energy spectrum $\sigma$ on a $L \times L$ comb array may be found by solving the eigenvalue equation [4, 6 ] :

$$
-t \sum_{x^{\prime}, y^{\prime}} A_{x, y ; x^{\prime}, y^{\prime}} \psi_{E}\left(x^{\prime}, y^{\prime}\right)=E \psi_{E}(x, y) .
$$

The spectrum $\sigma$ is formed by $L^{2}$ states and it is divided in four regions: $\left\{E_{0}\right\}, \sigma_{-}, \sigma_{0}$ and $\sigma_{+}[\underline{4}]$. $E_{0}$ is the ground-state energy which, with periodic boundary conditions and in the thermodynamic limit $L \rightarrow \infty$, is given by $E_{0}=-2 \sqrt{2} t$. $\sigma_{0}$ is the part of the spectrum corresponding to delocalized states with energies between $-2 t$ and $2 t$; the density of states of $\sigma_{0}$ is

$$
\rho(E)=\frac{1}{\pi \sqrt{4 t^{2}-E^{2}}}
$$




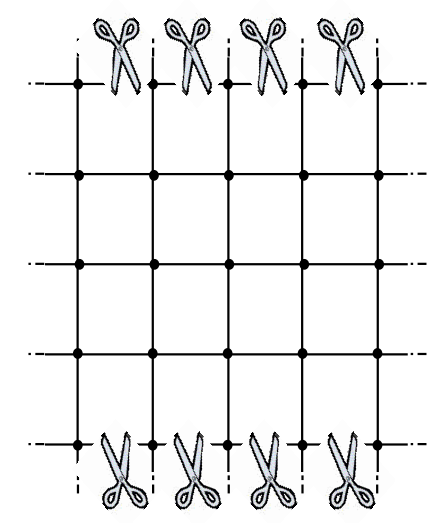

FIG. 2: From square lattice to comb.

just as for a particle hopping in a linear chain. The so-called hidden spectrum [4] is given by the union of $\sigma_{-}=$ $\left\{-2 t \sqrt{1+\cos ^{2}(k)}\right\}$ and $\sigma_{+}=\left\{2 t \sqrt{1+\cos ^{2}(k+\pi / 2)}\right\}$, where $k=2 \pi n / L$ is the wave vector along the backbone introduced by the Fourier transform of the $x$ coordinate and $n=1, \ldots,(L-1) / 4$; the hidden spectrum does not contribute to the normalized density of states in the thermodynamic limit since the number of these states is $L[\underline{4}]$. The ground-state and the eigenstates belonging to $\sigma_{-}$and $\sigma_{+}$- due to the particular topology of the array - are localized along the backbone and exhibit an exponential decay in the direction of the fingers. Omitting the normalization factor, the ground-state wavefunction is given by [4]

$$
\psi_{E_{0}}^{c o m b}(x, y)=e^{-|y| / \xi_{0}},
$$

where $\xi_{0}$ satisfies the transcendental equation $\sinh \left(1 / \xi_{0}\right)-\operatorname{coth}\left[L /\left(2 \xi_{0}\right)\right]=0$; the associated eigenvalue is $E_{0}=$ $-t\left(e^{-1 / \xi_{0}}+e^{1 / \xi_{0}}\right)$. As we shall see in the next section, bosons are allowed to spatially condense in this localized state.

It is instructive to provide a more intuitive path to evidence how a mere change in the topology of a network may give rise to the localized ground-state for bosons hopping on a comb graph. For this purpose one may start from a square lattice and imagine to systematically cut the bonds linking the sites of the square lattice in the $x$-direction at a chosen value of the $y$ coordinate (see Fig. 2); if one cuts all the bonds in the $x$ direction but the ones at the origin $y=0$, one obtains the comb lattice. Starting from a square lattice with $(2 M+1) \times(2 M+1)$ sites, the procedure to follow is to remove all the links in the $x$ direction at distance $|d|=M$ from the origin and then gradually cut stripes of links at $|d|=M-1, \ldots, 1$. As a result, one gets $M$ different arrays, each described by a different adjacency matrix $A_{x, y ; x^{\prime}, y^{\prime}}^{m}$, where $2 m$ is the number of cuts and $(2 M+1)-2 m \equiv 2 b+1$ is the number of remaining backbones $(b=M-m)$. For the square lattice one has:

$$
A_{x, y ; x^{\prime}, y^{\prime}}^{s q .}=\left(\delta_{x, x^{\prime}+1}+\delta_{x, x^{\prime}-1}\right) \cdot \delta_{y, y^{\prime}}+\delta_{x, x^{\prime}} \cdot\left(\delta_{y, y^{\prime}+1}+\delta_{y, y^{\prime}-1}\right),
$$

for a square with $2 m$ cuts the adjacency matrix is, for $m \geq 1$, given by

$$
A_{x, y ; x^{\prime}, y^{\prime}}^{m}=A_{x, y ; x^{\prime}, y^{\prime}}^{s q .}-2 \sum_{q=0}^{m-1}\left(\delta_{x, x^{\prime}+1}+\delta_{x, x^{\prime}-1}\right) \delta_{y, y^{\prime}} \delta_{y, M-q} .
$$

To determine the energy of the ground-state as a function of the number of cuts, it is most convenient to use a variational approach. For this purpose one may use a trial ground-state wavefunction $\psi_{\xi}(x, y)$ and then minimize the value of the energy $E(\xi, b ; M)$ with respect to the parameter $\xi$. With periodic boundary conditions, $\psi_{\xi}(x, y)$ should interpolate - as $m$ increases - between the ground-state wavefunction corresponding to bosons hopping on a square lattice (a constant with associated eigenvalue $E_{0}=-4 t$ ) and the wavefunction (7), which is the ground-state wavefunction for bosons hopping on a comb graph.

The simplest choice of the trial ground-state wavefunction taking into account both limits is given by

$$
\psi_{\xi}(x, y)=e^{-|y| / \xi},
$$




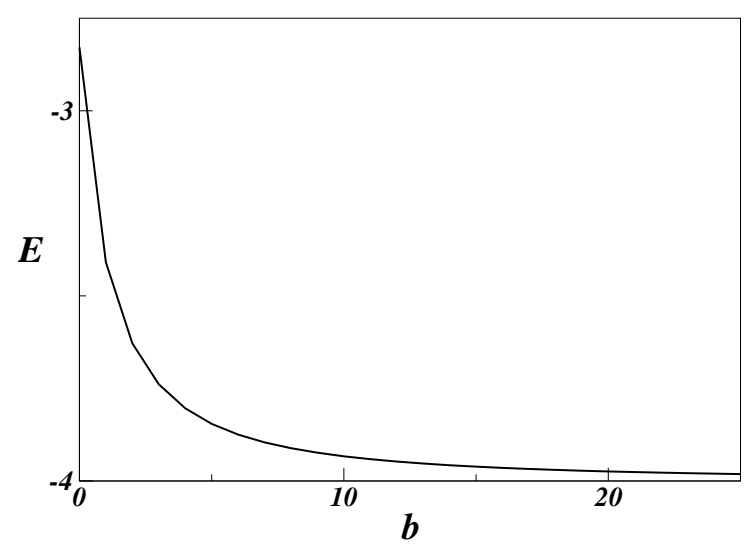

FIG. 3: Variational energy (13), in units of $t$, of the ground-state of the single-particle eigenvalue equation (5) as a function of the number of backbones $b$ in the thermodynamic limit $M \rightarrow \infty$. When $b=0$, one finds the ground-state energy of the comb graph $E_{0}=-2 \sqrt{2} t$.

where $\xi>0$ is the variational parameter. The variational energy of the ground-state of bosons hopping on a lattice with $2 m$ stripes of links removed is given by:

$$
E(\xi, m ; M)=-t \frac{\left\langle\psi_{\xi}\left|A^{m}\right| \psi_{\xi}\right\rangle}{\left\langle\psi_{\xi} \mid \psi_{\xi}\right\rangle}=-t \frac{\sum_{x, y=-M}^{M} \sum_{x^{\prime}, y^{\prime}=-M}^{M} \psi_{\xi}(x, y) A_{x, y ; x^{\prime}, y^{\prime}}^{m} \psi_{\xi}\left(x^{\prime}, y^{\prime}\right)}{\sum_{x, y=-M}^{M} \psi_{\xi}(x, y) \psi_{\xi}(x, y)} .
$$

As a function of the number of backbones, one has:

$$
E(b, \xi ; M)=t \frac{e^{-2 b / \xi}\left\{4 e^{(1+2 b) / \xi}+e^{2 M / \xi}\left[2-e^{-2 b / \xi}\left(1+e^{1 / \xi}\right)^{2}\right]\right\}}{e^{2 M / \xi}+e^{2(M+1) / \xi}-2} .
$$

If one minimizes Eq. (12) with respect to the parameter $\xi$, one obtains the variational ground-state wavefunction and its associated energy as a function of $b$.

The results are summarized in Figs. 35 and are compared with the results of a numerical evaluation of the groundstate wavefunction and its energy. In Fig. 3 we plot the variational energy in the thermodynamic limit which is taken by keeping $b$ (i.e., the number of remaining backbones) constant and performing the limit $M \rightarrow \infty$; the energy is given by

$$
E(b, \xi)=t \frac{2\left[2 e^{-2 b / \xi}-\left(1+e^{1 / \xi}\right)^{2}\right]}{1+e^{2 / \xi}} .
$$

As expected, effects due to the network topology clearly emerge if the number of remaining backbones is a zeromeasure set with respect to the number of stripes in a square lattice. In Fig. 4 we plot the numerical ground-state wavefunction of bosons hopping on a network with $(51 \times 51)$ sites for different values of $m$. Figure 4 evidences that the simplest trial wavefunction given in Eq. (10) is rather accurate in describing the shape of the exact wavefunction for a number of cuts $m \sim M$. In Fig. [5 we compare the energies pertinent to the ground-states discussed in this section: crosses correspond to the numerical results and the solid line to the ansatz (10). We also plot, as a function of the number of cuts $m$, the energy of the ground-state of bosons hopping on a square lattice: for $m=0$ the energy is $-4 t$, while for $m=M$ (corresponding to the comb lattice) is $-2 t$. The dashed line is the energy of the ground-state (7) of bosons hopping on a comb lattice as a function of the number of cuts $m$ : for $m=M$ the energy is $-2 \sqrt{2} t$, while for $m=0$ the wavefunction (7) is, of course, an excited state. Figure 5 clearly illustrates how the localized ground-state of bosons hopping on the comb becomes energetically more favorable when one increases the number of cuts.

The variational and numerical analysis carried out above provides us with an intuitive explanation of the appearance of localized states in the spectrum of a quantum particle hopping on a pertinent graph; furthermore, it clearly relates this property to the topology change of the network. 


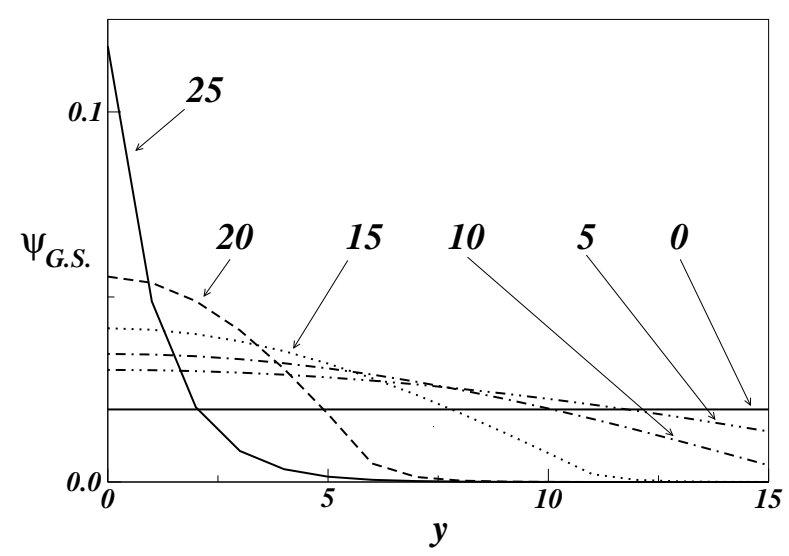

FIG. 4: The normalized single-particle ground-state wavefunction $\psi(x, y)$ computed numerically as a function of the distance $y$ from the backbone for a comb graph with $51 \times 51$ sites and different numbers of cuts $m=25,20,15,10,5$, and 0 . $m=0$ corresponds to the square lattice and $m=25$ to the comb.

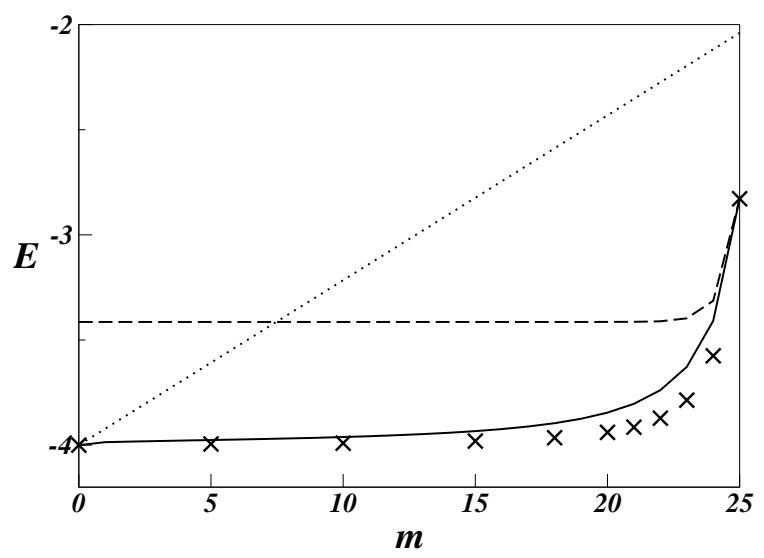

FIG. 5: Single-particle ground-state energies(in units of $t$ ) as a function of the number of cuts $m$ for a $51 \times 51$ lattice: crosses correspond to the numerical results, the solid line to the ansatz (10). We also plot the energies of the ground-state of bosons hopping on a square lattice (dotted line) and on a comb lattice (dashed line).

\section{THERMODYNAMICS OF BOSONS HOPPING ON A COMB GRAPH}

The thermodynamic properties of non-interacting bosons hopping on a comb graph hint to the possibility of a topology induced spatial BEC even if $d<2$ [4, 6]. To elucidate this new phenomenon, it is most convenient to introduce the macrocanonical ensemble to determine the fugacity $z=e^{\beta\left(\mu-E_{0}\right)}$ as a function of the temperature [4]: for a lattice with $L \times L$ sites, the equation determining $z$ is given by

$$
f L^{2} \equiv N_{T}=\sum_{E \in \sigma} \frac{d(E)}{z^{-1} e^{\beta(E+\sqrt{8} t)}-1} .
$$

In Eq. (14), $d(E)$ is the degeneracy of each single-particle eigenstate of the Hamiltonian (44) and $\beta=1 / k_{B} T$; the sum in Eq. (14) is over the entire spectrum $\sigma$. For free bosons hopping on a comb graph, one has [4, 6, 12]

$$
N_{T}=N_{E_{0}}(L, T)+N_{\sigma_{-}}(L, T)+N_{\sigma_{+}}(L, T)+\int_{E \in \sigma_{0}} d E \frac{L^{2} \rho(E)}{z^{-1} e^{\beta(E+\sqrt{8} t)}-1},
$$

where $N_{E_{0}}(L, T), N_{\sigma_{-}}(L, T)$ and $N_{\sigma_{+}}(L, T)$ denote, respectively, the number of particles at a certain temperature $T$ in the ground-state and in the two regions $\sigma_{-}$and $\sigma_{+}$of the hidden spectrum. $\rho(E)$, with $E \in \sigma_{0}$, is the energy 
density of states defined in Eq. (6), with $\sigma_{0}$ being the region of the spectrum corresponding to delocalized states. It is also useful to define the number of particles per site in each part of the spectrum as $n_{E_{0}}=N_{E_{0}} / L^{2}, n_{\sigma_{0}}=N_{\sigma_{0}} / L^{2}$, $n_{\sigma_{-}}=N_{\sigma_{-}} / L^{2}$ and $n_{\sigma_{+}}=N_{\sigma_{+}} / L^{2}$. In the thermodynamic limit, one has [12]

$$
\begin{gathered}
n_{E_{0}}(T)=\lim _{L \rightarrow \infty} \frac{1}{L^{2}} \frac{1}{z^{-1}-1}, \\
n_{\sigma_{-}}(T)=\lim _{L \rightarrow \infty} \frac{2}{L^{2}} \sum_{n=1}^{(L-1) / 4} \frac{1}{z^{-1} e^{\beta t\left[\sqrt{8}-2 \sqrt{1+\cos ^{2}(2 \pi n / L)}\right]}-1},
\end{gathered}
$$

and

$$
\begin{aligned}
n_{\sigma_{+}}(T) & =\lim _{L \rightarrow \infty} \frac{1}{L^{2}} \sum_{n=1}^{(L-1) / 4} \frac{2}{z^{-1} e^{\beta t\left[\sqrt{8}+2 \sqrt{1+\cos ^{2}(2 \pi n / L+\pi / 2)}\right]}-1} \\
& <\lim _{L \rightarrow \infty} \frac{2}{L} \frac{1}{z^{-1} e^{\beta t(\sqrt{8}+2)}-1}=0 \quad \forall T .
\end{aligned}
$$

Thus, in the thermodynamic limit, $\sigma_{+}$is not macroscopically occupied at any temperature and does not play any role in describing the thermodynamics of the system.

The last term of the right-hand side of Eq. (15) is the number of bosons in the delocalized (chain-like) states. The presence of the hidden spectrum changes the behavior of the integral evaluated in the interval $\{-2 t, 2 t\}$, since it reduces it to the one describing non-interacting bosons on a linear chain with an impurity in one of the sites. As a result, letting $z \rightarrow 1$, the integral converges even at finite temperatures making possible a spatial BEC in $d<2$.

\section{A. Critical temperature and condensate fraction}

If one defines $T_{c}$ as the critical temperature at which BEC occurs, for any $T<T_{c}$ the ground-state is macroscopically filled. Since, at the critical temperature, $n_{E_{0}}\left(T_{c}\right)=n_{\sigma_{ \pm}}\left(T_{c}\right)=0$, the equation allowing to determine $T_{c}$ as a function of the parameters $f$ and $t$ reads:

$$
\pi f=\int_{-2 t}^{2 t} \frac{d E}{\sqrt{4 t^{2}-E^{2}}} \frac{1}{e^{(E+\sqrt{8} t) /\left(k_{B} T_{c}\right)}-1} .
$$

Equation (16) can be solved numerically for any value of $f$. When $f \gg 1$, one may expand the exponential in Eq. (16) to the first order in the inverse of the critical temperature $T_{c}$ getting

$$
f \approx \int_{-2 t}^{2 t} d E \frac{1}{\pi \sqrt{4 t^{2}-E^{2}}} \frac{k_{B} T_{c}}{E+\sqrt{8} t}=\frac{k_{B} T_{c}}{2 t} .
$$

The critical temperature $T_{c}$ is then a linear function of both $t$ and $f$ given by:

$$
T_{c} \approx \frac{2 t f}{k_{B}} .
$$

Equation (18) has been checked numerically and it is in excellent agreement with the numerical solution of Eq. (16) for $f \gg 1$, the error being of order $1 / f$. By means of Eq. (3), one finds that the critical temperature for the occurrence of $\mathrm{BEC}$ is

$$
T_{c} \approx \frac{J_{0}}{k_{B}}
$$

One may now use Eq. (18) to determine the condensate fraction as a function of the scaled temperature $T / T_{c}$. In the thermodynamic limit, the number of particles in the delocalized states is given by

$$
N_{\sigma_{0}}\left(\frac{T}{T_{c}}\right)=\lim _{L \rightarrow \infty} L^{2} \int_{E \in \sigma_{0}} \rho(E) \frac{d E}{e^{\beta(E+\sqrt{8} t)}-1} \approx N_{T} \frac{T}{T_{c}} .
$$


In the last equation the exponential has been expanded to the first order in $\beta$ : this approximation holds for $f \gg 1$ and it is in very good agreement with the numerical evaluation of the integral in Eq. (20) also in a large neighborhood below $T_{c}$. From Eqs. (15) and (20) one gets the number of particles in the localized states $N_{0}=N_{E_{0}}+N_{\sigma_{-}}$: the fraction of condensate, for $T<T_{c}$, is then given by

$$
\frac{N_{0}}{N_{T}} \approx 1-\frac{T}{T_{c}} .
$$

For $f$ ranging from $10^{3}$ to $10^{9}$, the results provided by Eq. (21) differ from those obtained by the numerical evaluation of $N_{0}$ from Eq. (15) by less than 1\%. Equation (21) clearly shows that the condensate has dimension 1; cigar-shaped one-dimensional atomic Bose condensates support, in fact, a condensate fraction given by Eq. (21) [13, 14].

\section{B. Distribution of bosons along the fingers}

In the following we shall determine the distribution of the bosons over the comb graph, since - due to the spatial condensation - one expects an inhomogeneous distribution of the bosons along the fingers. The average number of bosons $N_{B}(x, y)$ on a site $(x, y)$ does not depend on $x$ - due to the translational invariance along the backbone - but only on the distance $y$ from the backbone, and - at any temperature $T$ - is given by:

$$
\begin{aligned}
N_{B}\left(y ; T / T_{c}\right) & =N_{E_{0}}\left(T / T_{c}\right)\left|\psi_{E_{0}}(y)\right|^{2}+\sum_{E_{n} \in \sigma_{-}} N_{\sigma_{-}}\left(E_{n} ; T / T_{c}\right)\left|\psi_{E_{n}}(y)\right|^{2} \\
& +L^{2} \int_{E \in \sigma_{0}} d E \rho(E) \frac{1}{e^{\beta(E+\sqrt{8} t)}-1}\left|\psi_{E}(y)\right|^{2} .
\end{aligned}
$$

In Eq. (22) $\psi_{E_{0}}(y)$ is the wavefunction corresponding to the ground-state of the single-particle spectrum and $\psi_{E_{n}}(y)$ are the eigenfunctions corresponding to the energies $E_{n}$ of the hidden spectrum $\sigma_{-} ; N_{\sigma_{-}}\left(E_{n}\right)$ is the number of particles with energies $E_{n} \in \sigma_{-}$and $N_{E_{0}}$ is the number of particles in the ground-state. In the last term of Eq. (22) $\psi_{E}(y)$ are the delocalized eigenfunctions of the eigenvalue equation (5).

To determine $N_{B}\left(y ; T / T_{c}\right)$, one needs an expression for $N_{E_{0}}$ and $N_{\sigma_{-}}$. For this purpose, it is useful to define the scaled temperature

$$
\tau=\frac{T}{T_{c}}
$$

For $\tau \leq 1$ and in the thermodynamic limit, $N_{\sigma_{-}}\left(E_{n}, \tau\right)$ is given by 12

$$
N_{\sigma_{-}}\left(E_{n} ; \tau\right)=\lim _{L \rightarrow \infty} L^{2} \frac{2}{\frac{2 \sqrt{2} t}{k_{B} T}(\pi n)^{2}+\frac{L^{2}}{N_{E_{0}}(\tau)}}
$$

and it depends on the number of particles in the ground-state $N_{E_{0}}$. Using the fact that $k_{B} T_{c} \approx 2 t f$, from Eq. (24) it follows that the number of particles in the hidden spectrum is given by

$$
N_{\sigma_{-}}(\tau)=\sum_{n=1}^{\infty} N_{\sigma_{-}}\left(E_{n}\right)=-N_{E_{0}}+N_{E_{0}} \sqrt{\frac{\tau}{\sqrt{2}} \frac{N_{T}}{N_{E_{0}}}} \operatorname{coth}\left[\sqrt{\frac{\tau}{\sqrt{2}} \frac{N_{T}}{N_{E_{0}}}}\right] .
$$

Requiring that $N_{0}=N_{\sigma_{-}}+N_{E_{0}}$ and using Eq. (21), one finds the way to determine $N_{E_{0}} / N_{T}$ as a function only of the scaled temperature $\tau$ :

$$
N_{T}(1-\tau)=N_{E_{0}} \sqrt{\frac{\tau}{\sqrt{2}} \frac{N_{T}}{N_{E_{0}}}} \operatorname{coth}\left[\sqrt{\frac{\tau}{\sqrt{2}} \frac{N_{T}}{N_{E_{0}}}}\right] .
$$

Solving Eq. (26) and substituting back the value obtained for $N_{E_{0}}$ in Eq. (24) allows for an exact numerical evaluation of Eq. (22).

Conventional wisdom supported by numerical evidence suggests however that - apart from a small range of temperatures near $T_{c}$ - the largest contribution to $N_{0}$ comes from $N_{E_{0}}$. Thus, it is physically appealing to assume the following form for $N_{E_{0}}$, namely,

$$
N_{E_{0}}=N_{T}(1-\tau) g(\tau)
$$




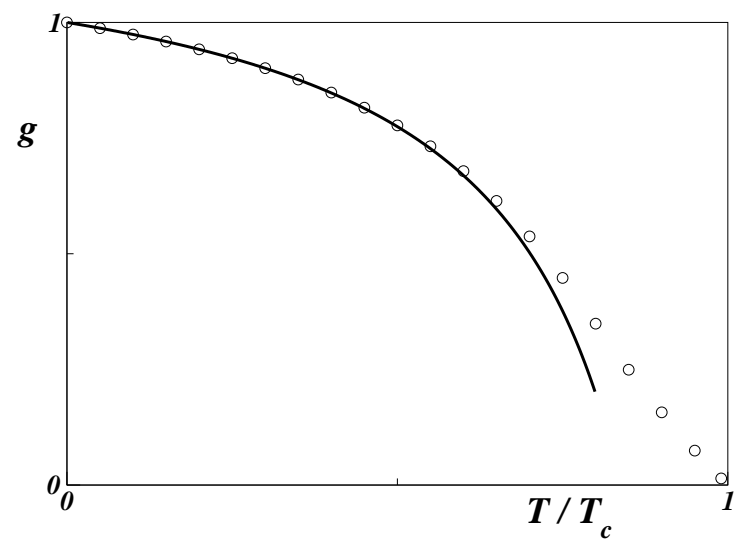

FIG. 6: The function $g\left(T / T_{c}\right)$ defined in Eq. 27): the empty circles correspond to the numerical solution of the self-consistency equation (28); the solid line corresponds to the approximate expression (29).

In Eq. (27), $g(\tau)$ is a function only of the scaled temperature $\tau$ and parametrizes the contributions to $N_{0}$ coming from the states belonging to the hidden spectrum: when $g=1$, the condensate is in the ground-state, while, for $g=0$, is in the states of the hidden spectrum. Substituting Eq. (27) in Eq. (24) and requiring $N_{0}=N_{\sigma_{-}}+N_{E_{0}}$ with $N_{0}$ given by Eq. (21), leads to a self-consistency equation for $g(\tau)$ :

$$
g(\tau) \sqrt{\frac{\tau}{\sqrt{2}(1-\tau) g(\tau)}} \operatorname{coth}\left[\sqrt{\frac{\tau}{\sqrt{2}(1-\tau) g(\tau)}}\right]=1
$$

For $\tau$ not too close to 1, a rather simple approximate solution of Eq. (28) is given by

$$
g(\tau) \approx 2-\sqrt{\frac{\tau}{\sqrt{2}(1-\tau)}} \operatorname{coth}\left[\sqrt{\frac{\tau}{\sqrt{2}(1-\tau)}}\right] .
$$

The error made in using Eq. (29) instead of the exact solution of Eq. (28) is within few percents: for $\tau \leq 0.5$ the error is less than $1 \%$, while for $\tau=0.7$ is about $5 \%$. In Fig. [6] we plot the function $g(\tau)$ as obtained from the numerical solution of the self-consistency Eq. (28) and from the approximate expression (29).

Upon inserting Eq. (29) in Eq. (27), one has

$$
\frac{N_{E_{0}}(\tau)}{N_{T}} \approx(1-\tau)\left(2-\sqrt{\frac{\tau}{\sqrt{2}(1-\tau)}} \operatorname{coth}\left[\sqrt{\frac{\tau}{\sqrt{2}(1-\tau)}}\right]\right)
$$

and, from Eq. (24), one gets

$$
\frac{N_{\sigma_{-}}(\tau)}{N_{T}}=\sum_{n=1}^{\infty} \frac{2}{\frac{\sqrt{2}(\pi n)^{2}}{\tau}+\frac{1}{(1-\tau) g(\tau)}} \approx(1-\tau)\left\{\sqrt{\frac{\tau}{\sqrt{2}(1-\tau)}} \operatorname{coth}\left[\sqrt{\frac{\tau}{\sqrt{2}(1-\tau)}}\right]-1\right\} .
$$

An explicit analytical form for the number of bosons at site $y, N_{B}(y)$, may now be derived. The last term in Eq. (22) gives, in fact, the contribution coming from the delocalized states: for a large network $(L \gg 1)$, and far away from the backbone, this number is independent from the site index $y$ and equals a constant $\left(N_{T} / L^{2}\right) \tau$. Then, using Eqs. (30) and (31), for $\tau<1$, one has

$$
\begin{aligned}
N_{B}(y ; \tau) & \approx \lim _{L \rightarrow \infty} N_{T}\left\{\frac { 1 } { L } \left[\left(1-\tau-\sum_{n=1}^{(L-1) / 4} \frac{2}{\frac{\sqrt{2}(\pi n)^{2}}{\tau}+\frac{1}{(1-\tau) g(\tau)}}\right) \frac{e^{-2 \operatorname{arcsinh}(1)|y|}}{\sqrt{2}}\right.\right. \\
& \left.\left.+\sum_{n=1}^{(L-1) / 4} \frac{2 \cos (2 \pi n / L)}{\frac{\sqrt{2}(\pi n)^{2}}{\tau}+\frac{1}{(1-\tau) g(\tau)}} \frac{e^{-2 \operatorname{arcsinh}[\cos (2 \pi n / L)]|y|}}{\sqrt{1+\cos ^{2}(2 \pi n / L)}}\right]+\frac{\tau}{L^{2}}\right\} .
\end{aligned}
$$


The exponential behavior typical of the eigenfunctions corresponding to the localized states leads, for $\tau<1$, to an increase of $N_{B}(y ; \tau)$ on the sites near the backbone while, when $y \gg 1$, the behavior is dominated by the last term in the right-hand side of Eq. (32). Thus, away from the backbone, once the filling is fixed, $N_{B}$ depends only on the scaled temperature $\tau$ and it is given by

$$
\frac{N_{B}\left(y ; T / T_{c}\right)}{f} \approx \tau \equiv \frac{T}{T_{c}}
$$

In the next section we shall show how this property may be useful to compute the observable effects induced by the existence of a spatial BEC in condensed matter systems.

\section{FURTHER OUTLOOK: SIGNATURE OF BEC FOR JOSEPHSON NETWORKS ON A COMB}

We proposed so far a theoretical framework establishing the role played by the network's topology in understanding the quantum and thermodynamic inhomogeneities arising in a system of bosons hopping on a comb graph; the approach enables one to obtain a simple and explicit analytical expression for the inhomogeneous distribution of bosons along the comb's fingers signaling the dramatic effect of the topology induced spatial BEC evidenced in Refs. [4, 6]. With little modifications our analysis could be carried out also for diverse network's topologies supporting BEC 6].

We shall now evidence how to detect the signature of BEC for a JJN built on a comb graph. For this purpose, one may think to perform a measurement of the $I-V$ characteristic of a single finger of the JJN and of the critical Josephson current along the finger. If one feeds, in fact, an external current $I_{e x t}$ at the extremities of the finger, one should expect to observe no voltage unless $I_{\text {ext }}$ is larger than the smallest of the critical currents of the junctions along the finger. Since, below $T_{c}$, the critical Josephson current of the finger is given by the smallest of the critical currents of the junctions positioned along the fingers, the measurement of the $I-V$ characteristic of the finger should provide a measurement of the critical current of the junctions at the top of the fingers (i.e., at $y \gg 1$ ).

To make a definite prediction, one needs to estimate the value of the Josephson critical current of a single junction of the network as a function of both the temperature and the distance from the backbone. Above $T_{c}$ the Cooper pairs, for a large array $(L \gg 1)$, are uniformly distributed over the network: the Josephson critical current is the same at each junction

$$
I_{c}^{A}(y) \approx t(4 e / \hbar) \cdot \sqrt{N_{B}(y+1) N_{B}(y)} \approx J_{0} \cdot(2 e / \hbar)
$$

where $I_{c}^{A}$ is the Josephson critical current above $T_{c}$. According to Eq. (3), the Josephson critical current depends only on the position and on the population of the sites.

The relation between the Josephson critical current of the junction between the sites $(x, y)$ and $(x, y+1)$ above and below the critical temperature $T_{c}$ does not depend on $x$ and it is given by

$$
\frac{I_{c}^{B}(y ; \tau)}{I_{c}^{A}(y)} \approx \frac{\sqrt{N_{B}(y+1, \tau) N_{B}(y, \tau)}}{f}
$$

where $I_{c}^{B}(y ; \tau)$ is the Josephson critical current below $T_{c}$ at distance $y$ from the backbone. $N_{B}(y, \tau)$ is given by Eq. (32). The result (35) - for a given $f$ - is independent from the total number of bosons in the system. Far from the backbone $(y \gg 1)$, one simply has

$$
\frac{I_{c}^{B}(y ; \tau)}{I_{c}^{A}(y)} \approx \frac{N_{B}(y, \tau)}{f}
$$

and, using Eq. (33), one gets

$$
\frac{I_{c}^{B}(y ; \tau)}{I_{c}^{A}(y)} \approx \tau \equiv \frac{T}{T_{c}}
$$

$I_{c}^{B}(y ; \tau)$ at the top of the finger can be experimentally measured as the critical Josephson current of the finger. In Fig 7 we plot Eq. (37).

BEC in a JJN built on a comb graph predicts then a rather sharp decrease of the Josephson critical current for a junction located away from the backbone and this behavior affects the measurement of the $I-V$ characteristic along a given finger of the JJN. The linear dependence exhibited by the solid line in Fig. 7 is consistent with the observation 


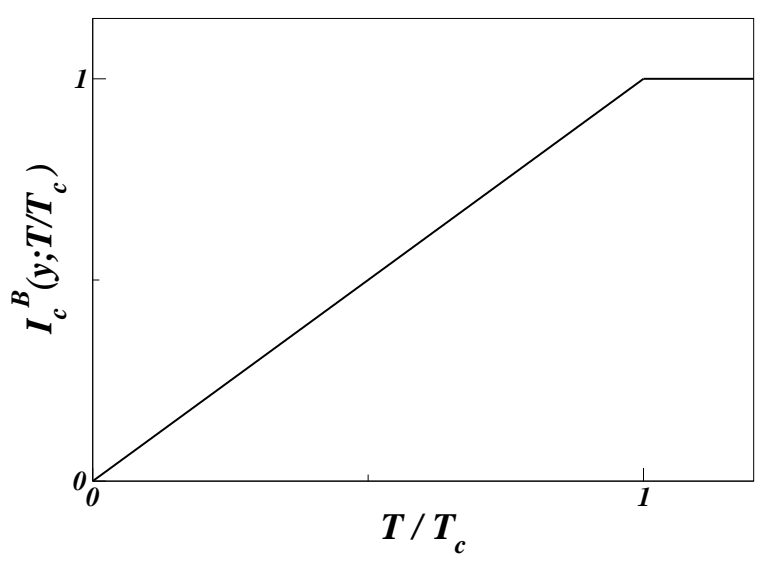

FIG. 7: Josephson critical current as a function of $T / T_{c}$ computed at distances from the backbone $y \gg 1 . I_{c}^{B}\left(y ; T / T_{c}\right)$ is in units of $I_{c}^{A}(y)$ (the critical current of the junctions above $T_{c}$ ) and is therefore equal to 1 for $T \geq T_{c}$.

that, in this system, the condensate has dimension 1. We stress that this phenomenon should be already observed for a classical JJN described by the Hamiltonian (1).

The slope of the linear plot in Fig. 7 provides a direct estimate for the fraction of condensate $N_{0} / N_{T} \approx 1-k_{B} T / J_{0}$ for a JJN built on a comb graph. In fact, far away from the backbone $(y \gg 1)$, from Eqs. (21), (36) and (37), one has that the fraction of condensate is given by $1-\left[I_{c}^{B}(y ; \tau) / I_{c}^{A}(y)\right]$.

To conclude this section, we notice that a similar analysis applies to a bosonic gas in comb-shaped deep optical lattice, provided that one defines the Josephson current of a single bosonic Josephson junction between two neighbor sites at $y$ and $y+1$ as $I_{c} \approx 2 t \sqrt{N_{B}(y) N_{B}(y+1)}$ (where $N_{B}(y)$ is the number of bosons in $y$ ). Above $T_{c}$, all the $N_{B}(y)$ will be equal to the filling $f$ at the equilibrium and $I_{c}^{A}=2 t f$. Below $T_{c}$, the equilibrium values for $N_{B}(y)$ change along the fingers according to Eq. (22) and the ratio between $I_{c}^{B}(y, \tau)$ and $I_{c}^{A}$ gives, for $y \gg 1$, directly the fraction of noncondensate atoms.

\section{Acknowledgements}

We thank Mario Rasetti, Matteo Cirillo, Carlo Cosmelli, and Alessandro Vezzani for the benefit of many stimulating discussions. We acknowledge financial support by M.I.U.R. through grant No. 2001028294. One of us (P.S.) would like to thank Francesco Guerra for his long-lasting friendship and continuous guidance since the years he was His student at the University of Naples.

[1] X. G. Wen, Phys. Lett. A 300, 175 (2002); Phys. Rev. B 65, 165113 (2003).

[2] X. G. Wen and Q. Niu, Phys. Rev. B 41, 9377 (1990).

[3] Y. Makhlin, G. Schön and A. Shnirman, Rev. Mod. Phys. 73, 357 (2001).

[4] R. Burioni, D. Cassi, I. Meccoli, M. Rasetti, S. Regina, P. Sodano, and A. Vezzani, Europhys. Lett. 52, 251 (2000).

[5] L. B. Ioffe and M. V. Feigel'man, Phys. Rev. B 66, 224503 (2002); B. Douçot, M. V. Feigel'man, and L. B. Ioffe, Phys. Rev. Lett. 90, 107003 (2003).

[6] R. Burioni, D. Cassi, M. Rasetti, P. Sodano, and A. Vezzani, J. Phys. B 34, 4697 (2001).

[7] R. Fazio and H. van der Zant, Phys. Rep. 355, 235 (2001).

[8] A. Barone and G. Paternò, Physics and applications of the Josephson effect, (Wiley and sons, New York, (1982).

[9] A. van Otterlo, K.-H. Wagenblast, R. Fazio and G. Schön, Phys. Rev. B 48, 3316 (1993).

[10] J. R. Anglin, P. Drummond, and A. Smerzi, Phys. Rev. A 64, 063605 (2001).

[11] S. Havlin and D. Ben-Avraham, Advances in Physics 51, 187 (2002). D. C. Mattis, Phys. Rev. B 20, 349 (1979); G. Weiss and S. Havlin, Physica A 134, 474, (1986).

[12] P. Buonsante, R. Burioni, D. Cassi, and A. Vezzani, Phys. Rev. B 66, 094207 (2002).

[13] W. Ketterle and N. J. van Druten, Phys. Rev. A 54, 656 (1996).

[14] A. Görlitz et al., Phys. Rev. Lett. 87, 130402 (2001). 